\title{
Electronic Processing of Antimicrobial Susceptibilities to Enhance Communicable Disease Surveillance
}

\author{
Emily Roberts \\ Informatics Program, Utah Department of Health, Salt Lake City, Utah, United States
}

Objective

Illustrate how the Utah Department of Health automatically processes antimicrobial susceptibility results that are received electronically

\section{Introduction}

The emerging threat of antimicrobial resistant organisms is a pressing public health concern. Surveillance for antimicrobial resistance can prevent infections, protect patients in the healthcare setting and improve antimicrobial use. In 2018, the Utah Department of Health mandated the reporting of antimicrobial susceptibility panels performed on selected organisms. Utah utilizes the Electronic Message Staging Area (EMSA), a home-grown application to translate, process, and enter electronic laboratory results into UT-NEDSS, Utah's integrated disease surveillance system. Processing these results electronically is challenging due to the need to interpret results based on the antimicrobial agent combined with the organism it was performed on. The receipt of antimicrobial susceptibility panels has required enhancements to EMSA for these results to be automatically processed.

\section{Methods}

Stand-alone antimicrobial susceptibility LOINCs are configured within EMSA to concatenate during the preprocessing stage. This tells EMSA that when this LOINC is sent within an HL7 message to find the organism name in the corresponding OBR 26.3 (the parent result field). EMSA then creates a new fabricated code that combines the antimicrobial agent with the organism identified from the culture (example: '18906-8 Pseudomonas aeruginosa' is the fabricated code for Ciprofloxacin susceptibility to Pseudomonas aeruginosa).

Once these new fabricated antimicrobial susceptibility codes are created, interpretation rules are programmed using current Clinical and Laboratory Standards Institute (CLSI) breakpoints for each unique organism/antimicrobial combination to determine if the result is Susceptible/Intermediate/Resistant. The interpreted test is then run through a set of condition-specific rules to determine how it should be included into UT-NEDSS.

\section{Results}

Antimicrobial susceptibility panels performed on Acinetobacter species, Escherichia coli, Klebsiella species, Pseudomonas aeruginosa, Enterobacter species, Candida auris/haemulonii, Mycobacterium tuberculosis, Neisseria gonorrhoeae, Salmonella species, Shigella species, Streptococcus pneumoniae and invasive Staphylococcus aureus are now included in Utah's Communicable Disease Reporting Rule. Currently, there are 36 antimicrobial agents programmed into EMSA and there are a total of 217 antimicrobial susceptibility codes programmed into the system.

\section{Conclusions}

Processing electronic antimicrobial susceptibility results presents unique challenges for processing. Interpretation of results can vary based on test method, performing laboratory, and organism. Enhancing functionality within EMSA was necessary for combining the antimicrobial agent and organism it was performed on. Implementing systems capable of automatically processing complicated antimicrobial susceptibility results should be a priority for any health department interested in expanding their communicable disease rule to include antimicrobial susceptibility testing. 